\title{
Differences in Designations of Observation Care in US Freestanding Children's Hospitals: Are They Virtual or Real?
}

\author{
Michelle L. Macy, MD, MS*, Matthew Hall, PhD², Samir S. Shah, MD, MSCE ${ }^{3}$, Carla Hronek, RN, MSN², \\ Mark A. Del Beccaro, MD ${ }^{4}$, Paul D. Hain, $\mathrm{MD}^{5}$, Elizabeth R. Alpern, MD, MSCE ${ }^{6}$
}

\begin{abstract}
${ }^{1}$ Department of Emergency Medicine and the Child Health Evaluation and Research (CHEAR) Unit, Division of General Pediatrics, University of Michigan, Ann Arbor, Michigan; 'Child Health Corporation of America, Shawnee Mission, Kansas; ${ }^{3}$ Division of Infectious Diseases, The Children's Hospital of Philadelphia, Department of Pediatrics, and Center for Clinical Epidemiology and Biostatistics, University of Pennsylvania School of Medicine, Philadelphia, Pennsylvania; ${ }^{4}$ Department of Pediatrics, University of Washington School of Medicine, Seattle Children's, Seattle, Washington; ${ }^{5}$ Department of Pediatrics, Vanderbilt University School of Medicine, Nashville, Tennessee; ${ }^{6}$ Division of Emergency Medicine, The Children's Hospital of Philadelphia, Department of Pediatrics, University of Pennsylvania School of Medicine, Philadelphia, Pennsylvania.
\end{abstract}

OBJECTIVE: To characterize practices related to observation care and to examine the current models of pediatric observation medicine in US children's hospitals.

DESIGN: We utilized 2 web-based surveys to examine observation care in the 42 hospitals participating in the Pediatric Health Information System database. We obtained information regarding the designation of observation status, including the criteria used to admit patients into observation. From hospitals reporting the use of observation status, we requested specific details relating to the structures of observation care and the processes of care for observation patients following emergency department treatment.

RESULTS: A total of 37 hospitals responded to Survey 1, and 20 hospitals responded to Survey 2. Designated observation units were present in only 12 of 31 (39\%) hospitals that report observation patient data to the Pediatric Health Information System. Observation status was variably defined in terms of duration of treatment and prespecified criteria. Observation periods were limited to $<48$ hours in 24 of 31 (77\%) hospitals. Hospitals reported that various standards were used by different payers to determine observation status reimbursement. Observation care was delivered in a variety of settings. Most hospitals indicated that there were no differences in the clinical care delivered to virtual observation status patients when compared with other inpatients.

CONCLUSIONS: Observation is a variably applied patient status, defined differently by individual hospitals. Consistency in the designation of patients under observation status among hospitals and payers may be necessary to compare quality outcomes and costs, as well as optimize models of pediatric observation care.Journal of Hospital Medicine 2012;7:287-293. (C) 2011 Society of Hospital Medicine.
Observation medicine has grown in recent decades out of changes in policies for hospital reimbursement, requirements for patients to meet admission criteria to qualify for inpatient admission, and efforts to avoid "unnecessary" or "inappropriate" admissions. ${ }^{1}$ Emergency physicians are frequently faced with patients who are too sick to be discharged home, but do not clearly meet criteria for an inpatient status admission. These patients often receive extended outpatient services (typically extending 24 to 48 hours) under the designation of observation status, in order to determine their response to treatment and need for hospitalization.

Observation care delivered to adult patients has increased substantially in recent years, and the confusion around the designation of observation versus

\footnotetext{
*Address for correspondence and reprint requests: Michelle L. Macy, MD, MS, Division of General Pediatrics, 300 North Ingalls 6C13, University of Michigan, Ann Arbor, Ml 48109-5456; Tel.: 734-936-8338; Fax: 734-764-2599; E-mail: mlmacy@umich.edu

Additional Supporting Information may be found in the online version of this article.

Received: November 15, 2010; Revised: May 3, 2011; Accepted: May 8,2011

2011 Society of Hospital Medicine DOI 10.1002/jhm.949

Published online in Wiley Online Library (Wileyonlinelibrary.com).
}

inpatient care has received increasing attention in the lay press. $^{2-7}$ According to the Centers for Medicare and Medicaid Services $(\mathrm{CMS})^{8}$ :

Observation care is a well-defined set of specific, clinically appropriate services, which include ongoing short term treatment, assessment, and reassessment before a decision can be made regarding whether patients will require further treatment as hospital inpatients.... Observation services are commonly ordered for patients who present to the emergency department and who then require a significant period of treatment or monitoring in order to make a decision concerning their admission or discharge.

Observation status is an administrative label that is applied to patients who do not meet inpatient level of care criteria, as defined by third parties such as InterQual $^{\circledR}$. These criteria usually include a combination of the patient's clinical diagnoses, severity of illness, and expected needs for monitoring and interventions, in order to determine the admission status to which the patient may be assigned (eg, observation, inpatient, or intensive care). Observation services can be provided, in a variety of settings, to those patients who do not meet inpatient level of care but require a period of observation. Some hospitals provide observation care in discrete units in the emergency department (ED) or specific inpatient unit, and others have no designated 
unit but scatter observation patients throughout the institution, termed "virtual" observation units."

For more than 30 years, observation unit (OU) admission has offered an alternative to traditional inpatient hospitalization for children with a variety of acute conditions. ${ }^{10,11}$ Historically, the published literature on observation care for children in the United States has been largely based in dedicated emergency department OUs. ${ }^{12}$ Yet, in a 2001 survey of 21 pediatric EDs, just 6 reported the presence of a "23-hour unit."13 There are single-site examples of observation care delivered in other settings. ${ }^{14,15}$ In 2 national surveys of US General Hospitals, $25 \%$ provided observation services in beds adjacent to the ED, and the remainder provided observation services in hospital inpatient units. ${ }^{16,17}$ However, we are not aware of any previous multi-institution studies exploring hospitalwide practices related to observation care for children.

Recognizing that observation status can be designated using various standards, and that observation care can be delivered in locations outside of dedicated OUs, ${ }^{9}$ we developed 2 web-based surveys to examine the current models of pediatric observation medicine in US children's hospitals. We hypothesized that observation care is most commonly applied as a billing designation and does not necessarily represent care delivered in a structurally or functionally distinct OU, nor does it represent a difference in care provided to those patients with inpatient designation.

\section{METHODS}

\section{Study Design}

Two web-based surveys were distributed, in April 2010, to the 42 freestanding, tertiary care children's hospitals affiliated with the Child Health Corporation of America (CHCA; Shawnee Mission, KS) which contribute data to the Pediatric Health Information System (PHIS) database. The PHIS is a national administrative database that contains resource utilization data from participating hospitals located in noncompeting markets of 27 states plus the District of Columbia. These hospitals account for $20 \%$ of all tertiary care children's hospitals in the United States.

\section{Survey Content Survey 1}

A survey of hospital observation status practices has been developed by CHCA as a part of the PHIS data quality initiative (see Supporting Appendix: Survey 1 in the online version of this article). Hospitals that did not provide observation patient data to PHIS were excluded after an initial screening question. This survey obtained information regarding the designation of observation status within each hospital. Hospitals provided free-text responses to questions related to the criteria used to define observation, and to admit patients into observation status. Fixed-choice response questions were used to determine specific observation status utilization criteria and clinical guidelines (eg, InterQual and Milliman ${ }^{\circledR}$ ) used by hospitals for the designation of observation status to patients.

\section{Survey 2}

We developed a detailed follow-up survey in order to characterize the structures and processes of care associated with observation status (see Supporting Appendix: Survey 2 in the online version of this article). Within the follow-up survey, an initial screening question was used to determine all types of patients to which observation status is assigned within the responding hospitals. All other questions in Survey 2 were focused specifically on those patients who required additional care following ED evaluation and treatment. Fixed-choice response questions were used to explore differences in care for patients under observation and those admitted as inpatients. We also inquired of hospital practices related to "boarding" of patients in the ED while awaiting admission to an inpatient bed.

\section{Survey Distribution}

Two web-based surveys were distributed to all 42 CHCA hospitals that contribute data to PHIS. During the month of April 2010, each hospital's designated PHIS operational contact received e-mail correspondence requesting their participation in each survey. Within hospitals participating in PHIS, Operational Contacts have been assigned to serve as the day-today PHIS contact person based upon their experience working with the PHIS data. The Operational Contacts are CHCA's primary contact for issues related to the hospital's data quality and reporting to PHIS. Non-responders were contacted by e-mail for additional requests to complete the surveys. Each e-mail provided an introduction to the topic of the survey and a link to complete the survey. The e-mail requesting participation in Survey 1 was distributed the first week of April 2010, and the survey was open for responses during the first 3 weeks of the month. The e-mail requesting participation in Survey 2 was sent the third week of April 2010, and the survey was open for responses during the subsequent 2 weeks.

\section{DATA ANALYSIS}

Survey responses were collected and are presented as a descriptive summary of results. Hospital characteristics were summarized with medians and interquartile ranges for continuous variables, and with percents for categorical variables. Characteristics were compared between hospitals that responded and those that did not respond to Survey 2 using Wilcoxon rank-sum tests and chi-square tests as appropriate. All analyses were performed using SAS v.9.2 (SAS Institute, Cary, NC), and a $P$ value $<0.05$ was considered statistically significant. The study was reviewed by the University of 


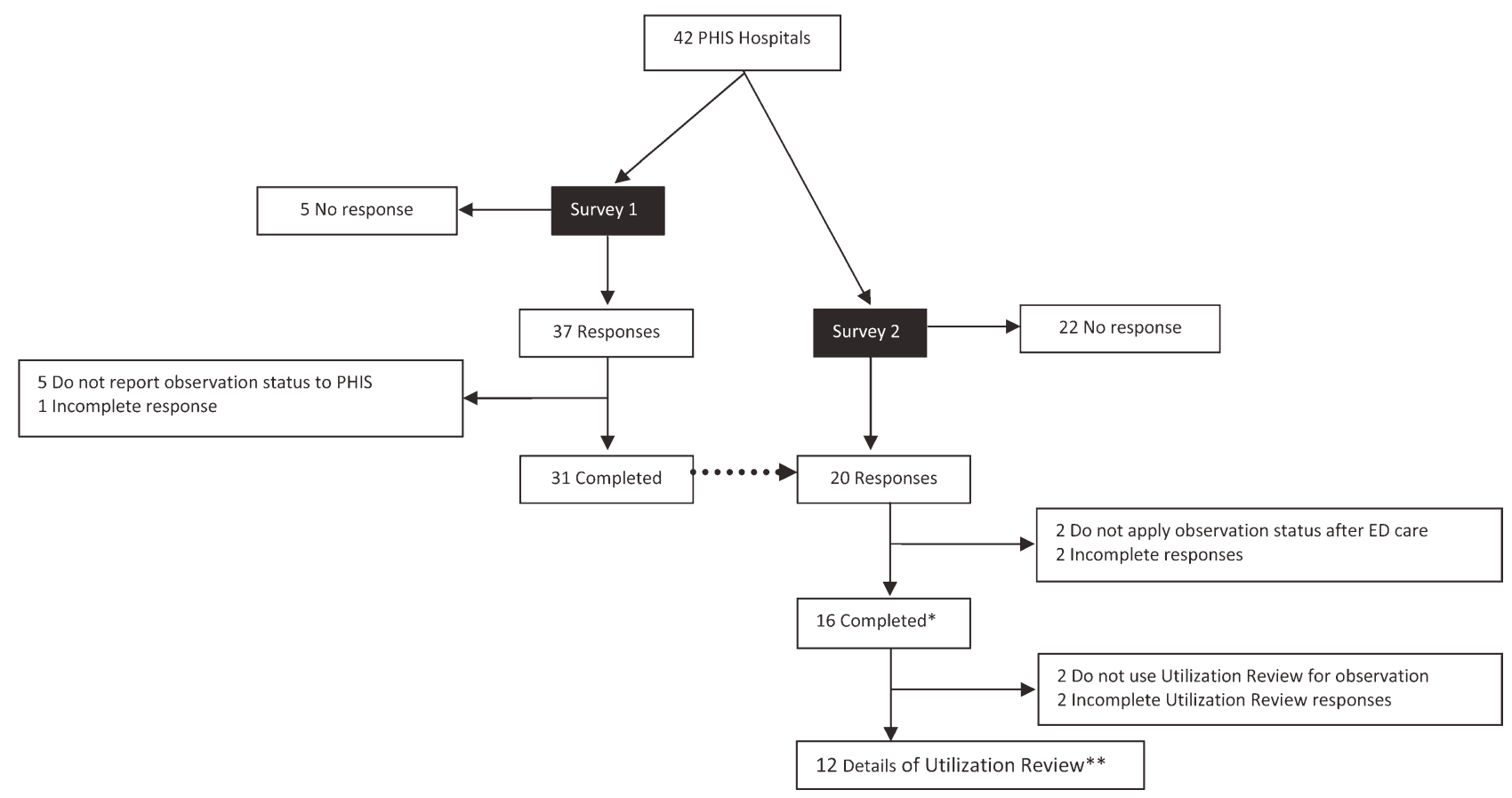

FIG. 1. Hospital responses to Survey 1 and Survey 2; exclusions and incomplete responses are included. Data from Survey 1 and Survey 2 could be linked for 17 hospitals. *Related data presented in Table 2. ${ }^{*}$ Related data presented in Table 3. Abbreviations: ED, emergency department; PHIS, Pediatric Health Information System.

\begin{tabular}{|c|c|c|c|}
\hline & Respondent $\mathrm{N}=20$ & Non-Respondent $\mathrm{N}=22$ & $P$ Value \\
\hline No. of inpatient beds Median [IQR] (excluding Obstetrics) & 245 [219-283] & 282 [250-381] & 0.076 \\
\hline Annual admissions Median [lQR] (excluding births) & $11,658[8,642-13,213]$ & $13,522[9,830-18,705]$ & 0.106 \\
\hline ED volume Median [lQR] & $60,528[47,850-82,955]$ & $64,486[47,386-84,450]$ & 0.640 \\
\hline Percent government payer Median [QR] & $53 \%[46-62]$ & $49 \%[41-58]$ & 0.528 \\
\hline \multicolumn{4}{|l|}{ Region } \\
\hline Northeast & $37 \%$ & $0 \%$ & 0.021 \\
\hline Midwest & $21 \%$ & $33 \%$ & \\
\hline South & $21 \%$ & $50 \%$ & \\
\hline West & $21 \%$ & $17 \%$ & \\
\hline Reports observation status patients to PHIS & $85 \%$ & $90 \%$ & 0.555 \\
\hline
\end{tabular}

Michigan Institutional Review Board and considered exempt.

\section{RESULTS}

Responses to Survey 1 were available from 37 of 42 $(88 \%)$ of PHIS hospitals (Figure 1). For Survey 2, we received responses from 20 of $42(48 \%)$ of PHIS hospitals. Based on information available from Survey 1, we know that 20 of the $31(65 \%)$ PHIS hospitals that report observation status patient data to PHIS responded to Survey 2. Characteristics of the hospitals responding and not responding to Survey 2 are presented in Table 1. Respondents provided hospital identifying information which allowed for the linkage of data, from Survey 1, to 17 of the 20 hospitals responding to Survey 2. We did not have information available to link responses from 3 hospitals.

Based on responses to the surveys and our knowledge of data reported to PHIS, our current understanding of patient flow from ED through observation to discharge home, and the application of observation status to the encounter, is presented in Figure 2. According to free-text responses to Survey 1, various methods were applied to designate observation status (gray shaded boxes in Figure 2). Fixedchoice responses to Survey 2 revealed that observation status patients were cared for in a variety of locations within hospitals, including ED beds, designated observation units, and inpatient beds (dashed boxes in Figure 2). Not every facility utilized all of the listed locations for observation care. Space constraints could dictate the location of care, regardless of patient status (eg, observation vs inpatient), in hospitals with more than one location of care available to observation patients. While patient status could change during a visit, only the final patient status at discharge enters the administrative record submitted to PHIS (black boxes in Figure 2). Facility charges for 


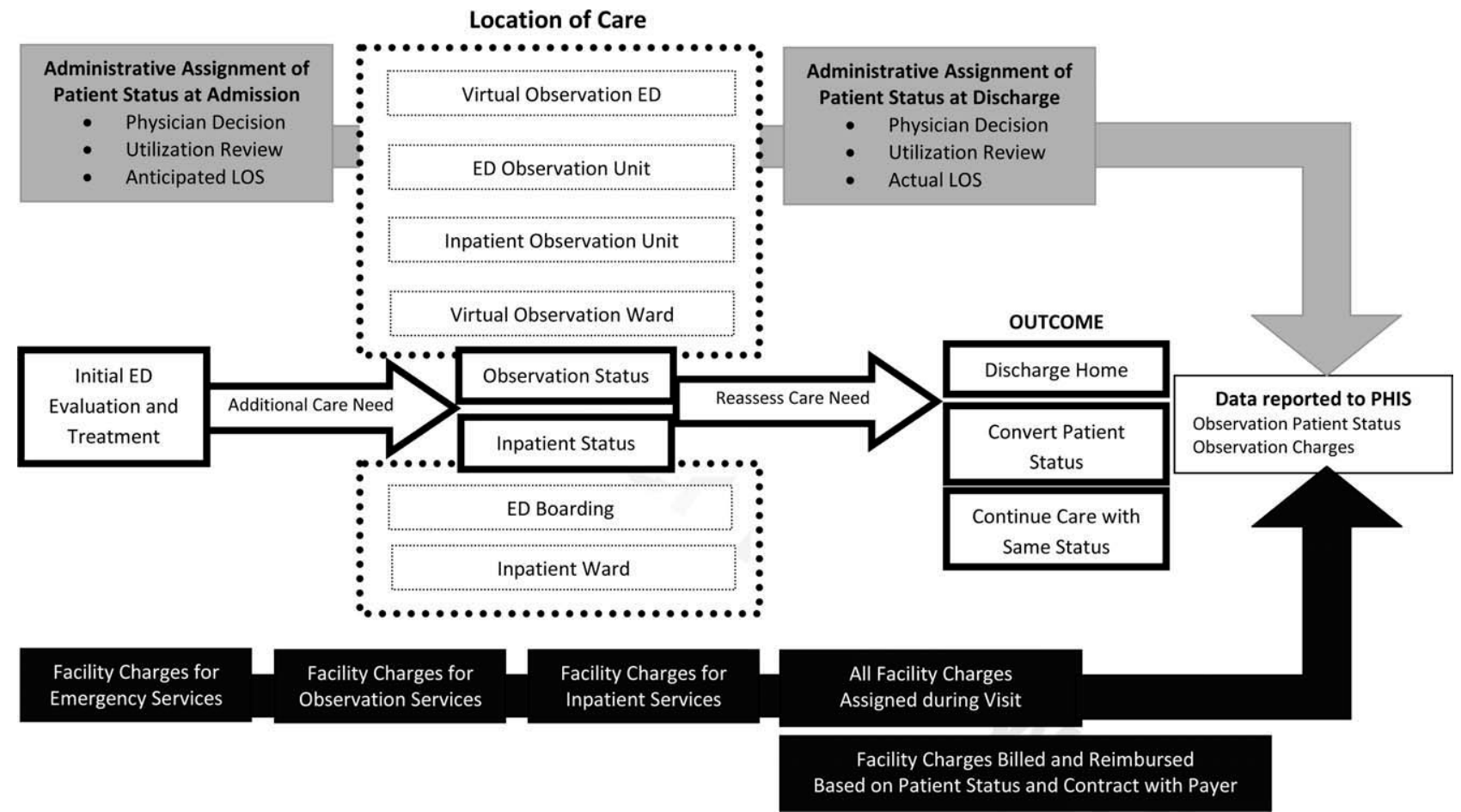

FIG. 2. Patient flow related to observation following emergency department care. The dashed boxes represent physical structures associated with observation and inpatient care that follow treatment in the ED. The gray shaded boxes indicate the points in care, and the factors considered, when assigning observation status. The black boxes show the assignment of facility charges for services rendered during each visit. Abbreviations: ED, emergency department; LOS, length of stay; PHIS, Pediatric Health Information System.

observation remained a part of the visit record and were reported to PHIS. Hospitals may or may not bill for all assigned charges depending on patient status, length of stay, or other specific criteria determined by contracts with individual payers.

\section{Survey 1: Classification of Observation Patients and Presence of Observation Units in PHIS Hospitals}

According to responses to Survey 1, designated OUs were not widespread, present in only 12 of the 31 hospitals. No hospital reported treating all observation status patients exclusively in a designated OU. Observation status was defined by both duration of treatment and either level of care criteria or clinical care guidelines in 21 of the 31 hospitals responding to Survey 1 . Of the remaining 10 hospitals, 1 reported that treatment duration alone defines observation status, and the others relied on prespecified observation criteria. When considering duration of treatment, hospitals variably indicated that anticipated or actual lengths of stay were used to determine observation status. Regarding the maximum hours a patient can be observed, 12 hospitals limited observation to 24 hours or fewer, 12 hospitals observed patients for no more than 36 to 48 hours, and the remaining 7 hospitals allowed observation periods of 72 hours or longer.

When admitting patients to observation status, 30 of 31 hospitals specified the criteria that were used to determine observation admissions. InterQual criteria, the most common response, were used by 23 of the
30 hospitals reporting specified criteria; the remaining 7 hospitals had developed hospital-specific criteria or modified existing criteria, such as InterQual or Milliman, to determine observation status admissions. In addition to these criteria, 11 hospitals required a physician order for admission to observation status. Twenty-four hospitals indicated that policies were in place to change patient status from observation to inpatient, or inpatient to observation, typically through processes of utilization review and application of criteria listed above.

Most hospitals indicated that they faced substantial variation in the standards used from one payer to another when considering reimbursement for care delivered under observation status. Hospitals noted that duration-of-care-based reimbursement practices included hourly rates, per diem, and reimbursement for only the first 24 or 48 hours of observation care. Hospitals identified that payers variably determined reimbursement for observation based on InterQual level of care criteria and Milliman care guidelines. One hospital reported that it was not their practice to bill for the observation bed.

\section{Survey 2: Understanding Observation Patient Type Administrative Data Following ED Care Within PHIS Hospitals}

Of the 20 hospitals responding to Survey 2, there were 2 hospitals that did not apply observation status to patients after ED care and 2 hospitals that did not 


\begin{tabular}{|c|c|c|c|c|c|c|}
\hline \multirow[b]{2}{*}{ Hospital No. } & \multirow{2}{*}{$\begin{array}{l}\text { Available Observation } \\
\text { Settings }\end{array}$} & \multicolumn{3}{|c|}{ Patient Groups Under Observation in Each Setting } & \multirow{2}{*}{$\begin{array}{l}\text { UR to Assign } \\
\text { Obs Status }\end{array}$} & \multirow{2}{*}{$\begin{array}{l}\text { When Obs Status } \\
\text { Is Assigned }\end{array}$} \\
\hline & & ED & Post-Op & Test/Treat & & \\
\hline \multirow[t]{2}{*}{1} & Virtual inpatient & $X$ & $X$ & $X$ & Yes & Discharge \\
\hline & Ward-based OU & & $x$ & $x$ & № & \\
\hline \multirow[t]{2}{*}{2} & Virtual inpatient & & $x$ & $x$ & Yes & Admission \\
\hline & Ward-based OU & $X$ & $X$ & $X$ & № & \\
\hline \multirow[t]{4}{*}{3} & Virtual inpatient & $x$ & $x$ & $x$ & Yes & Discharge \\
\hline & Ward-based OU & $x$ & $x$ & $X$ & Yes & \\
\hline & ED OU & $x$ & & & Yes & \\
\hline & Virtual ED & $x$ & & & Yes & \\
\hline \multirow[t]{3}{*}{4} & Virtual inpatient & $x$ & $x$ & $x$ & Yes & Discharge \\
\hline & ED OU & $x$ & & & № & \\
\hline & Virtual ED & $x$ & & & No & \\
\hline 5 & Virtual inpatient & $x$ & $x$ & $X$ & $N / A$ & Discharge \\
\hline 6 & Virtual inpatient & $x$ & $x$ & $x$ & Yes & Discharge \\
\hline \multirow[t]{3}{*}{7} & Virtual inpatient & $X$ & $x$ & & Yes & No response \\
\hline & Ward-based OU & $x$ & & & Yes & \\
\hline & Virtual ED & $x$ & & & Yes & \\
\hline 8 & Virtual inpatient & $x$ & $x$ & $X$ & Yes & Admission \\
\hline \multirow[t]{3}{*}{9} & Virtual inpatient & $x$ & $x$ & & Yes & Discharge \\
\hline & ED OU & $x$ & & & Yes & \\
\hline & Virtual ED & $X$ & & & Yes & \\
\hline \multirow[t]{2}{*}{10} & Virtual inpatient & $x$ & $x$ & $x$ & Yes & Admission \\
\hline & ED OU & $X$ & & & Yes & \\
\hline \multirow[t]{4}{*}{11} & Virtual inpatient & & $x$ & $x$ & Yes & Discharge \\
\hline & Ward-based OU & & $x$ & $x$ & Yes & \\
\hline & ED OU & $x$ & & & Yes & \\
\hline & Virtual ED & $X$ & & & Yes & \\
\hline 12 & Virtual inpatient & $x$ & $x$ & $x$ & Yes & Admission \\
\hline \multirow[t]{2}{*}{13} & Virtual inpatient & & $x$ & $x$ & $N / A$ & Discharge \\
\hline & Virtual ED & $x$ & & & $N / A$ & \\
\hline 14 & Virtual inpatient & $x$ & $X$ & $X$ & Yes & Both \\
\hline \multirow[t]{2}{*}{15} & Virtual inpatient & $x$ & $x$ & & Yes & Admission \\
\hline & Ward-based OU & $x$ & $x$ & & Yes & \\
\hline 16 & Virtual inpatient & $x$ & & & Yes & Admission \\
\hline
\end{tabular}

Abbreviations: ED, emergency department; N/A, not available; Obs, observation; OU, observation unit; Post-Op, postoperative care following surgery or procedures, such as tonsillectomy or cardiac catheterization; Test/Treat, scheduled tests and treatments such as EEG monitoring and infusions; UR, utilization review.

provide complete responses. The remaining 16 hospitals provided information regarding observation status as applied to patients after receiving treatment in the ED. The settings available for observation care and patient groups treated within each area are presented in Table 2. In addition to the patient groups listed in Table 2, there were 4 hospitals where patients could be admitted to observation status directly from an outpatient clinic. All responding hospitals provided virtual observation care (ie, observation status is assigned but the patient is cared for in the existing ED or inpatient ward). Nine hospitals also provided observation care within a dedicated ED or ward-based OU (ie, a separate clinical area in which observation patients are treated).

When asked to identify differences between clinical care delivered to patients admitted under virtual observation and those admitted under inpatient status, 14 of 16 hospitals selected the option "There are no differences in the care delivery of these patients." The differences identified by 2 hospitals included patient care orders, treatment protocols, and physician documentation. Within the hospitals that reported utilization of virtual ED observation, 2 reported differences in care compared with other ED patients, including patient care orders, physician rounds, documentation, and discharge process. When admitted patients were boarded in the ED while awaiting an inpatient bed, 11 of 16 hospitals allowed for observation or inpatient level of care to be provided in the ED. Fourteen hospitals allow an admitted patient to be discharged home from boarding in the ED without ever receiving care in an inpatient bed. The discharge decision was made by ED providers in 7 hospitals, and inpatient providers in the other 7 hospitals.

Responses to questions providing detailed information on the process of utilization review were provided by 12 hospitals. Among this subset of hospitals, utilization review was consistently used to assign virtual inpatient observation status and was applied at admission $(\mathrm{n}=6)$ or discharge $(\mathrm{n}=8)$, depending on the hospital. One hospital applied observation status at both admission and discharge; 1 hospital did not provide a response. Responses to questions regarding utilization review are presented in Table 3. 
TABLE 3. Utilization Review Practices Related to Observation Status

\begin{tabular}{lrl}
\hline Survey Question & Yes N (\%) & No N (\%) \\
\hline Preadmission utilization review is conducted at my hospital. & $3(25)$ & $9(75)$ \\
Utilization review occurs daily at my hospital. & $10(83)$ & $2(17)$ \\
A nonclinician can initiate an order for observation status. & $4(33)$ & $8(67)$ \\
Status can be changed after the patient has been discharged. & $10(83)$ & $2(17)$ \\
Inpatient status would always be assigned to a patient who & $9(75)$ & $3(25)$ \\
$\quad$ receives less than 24 hours of care and meets inpatient criteria. & & \\
The same status would be assigned to different patients who & $6(50)$ & $6(50)$ \\
$\quad$ received the same treatment of the same duration & & \\
but have different payers. & &
\end{tabular}

\section{DISCUSSION}

This is the largest descriptive study of pediatric observation status practices in US freestanding children's hospitals and, to our knowledge, the first to include information about both the ED and inpatient treatment environments. There are two important findings of this study. First, designated OUs were uncommon among the group of freestanding children's hospitals that reported observation patient data to PHIS in 2010. Second, despite the fact that hospitals reported observation care was delivered in a variety of settings, virtual inpatient observation status was nearly ubiquitous. Among the subset of hospitals that provided information about the clinical care delivered to patients admitted under virtual inpatient observation, hospitals frequently reported there were no differences in the care delivered to observation patients when compared with other inpatients.

The results of our survey indicate that designated OUs are not a commonly available model of observation care in the study hospitals. In fact, the vast majority of the hospitals used virtual inpatient observation care, which did not differ from the care delivered to a child admitted as an inpatient. ED-based OUs, which often provide operationally and physically distinct care to observation patients, have been touted as cost-effective alternatives to inpatient care ${ }^{18-20}$ resulting in fewer admissions and reductions in length of stay ${ }^{19,20}$ without a resultant increase in return EDvisits or readmissions. ${ }^{21-23}$ Research is needed to determine the patient-level outcomes for short-stay patients in the variety of available treatment settings (eg, physically or operationally distinct OUs and virtual observation), and to evaluate these outcomes in comparison to results published from designated OUs. The operationally and physically distinct features of a designated OU may be required to realize the benefits of observation attributed to individual patients.

While observation care has been historically provided by emergency physicians, there is increasing interest in the role of inpatient providers in observation care. ${ }^{9}$ According to our survey, children were admitted to observation status directly from clinics, following surgical procedures, scheduled tests and treatment, or after evaluation and treatment in the ED. As many of these children undergo virtual observation in inpatient areas, the role of inpatient providers, such as pediatric hospitalists, in observation care may be an important area for future study, education, and professional development. Novel models of care, with hospitalists collaborating with emergency physicians, may be of benefit to the children who require observation following initial stabilization and treatment in the ED. ${ }^{24,25}$

We identified variation between hospitals in the methods used to assign observation status to an episode of care, including a wide range of length of stay criteria and different approaches to utilization review. In addition, the criteria payers use to reimburse for observation varied between payers, even within individual hospitals. The results of our survey may be driven by issues of reimbursement and not based on a model of optimizing patient care outcomes using designated OUs. Variations in reimbursement may limit hospital efforts to refine models of observation care for children. Designated OUs have been suggested as a method for improving ED patient flow, ${ }^{26}$ increasing inpatient capacity, ${ }^{27}$ and reducing costs of care. ${ }^{28}$ Standardization of observation status criteria and consistent reimbursement for observation services may be necessary for hospitals to develop operationally and physically distinct OUs, which may be essential to achieving the proposed benefits of observation medicine on costs of care, patient flow, and hospital capacity.

\section{LIMITATIONS}

Our study results should be interpreted with the following limitations in mind. First, the surveys were distributed only to freestanding children's hospitals who participate in PHIS. As a result, our findings may not be generalizable to the experiences of other children's hospitals or general hospitals caring for children. Questions in Survey 2 were focused on understanding observation care, delivered to patients following ED care, which may differ from observation practices related to a direct admission or following scheduled procedures, tests, or treatments. It is important to note that, hospitals that do not report observation status patient data to PHIS are still providing care to children with acute conditions that respond to brief periods of hospital treatment, even though it is not labeled observation. However, it was beyond the scope of this study to characterize the care delivered to all patients who experience a short stay.

The second main limitation of our study is the lower response rate to Survey 2. In addition, several surveys contained incomplete responses which further limits our sample size for some questions, specifically those related to utilization review. The lower response to Survey 2 could be related to the timing of the distribution of the 2 surveys, or to the information contained in the introductory e-mail describing Survey 2. Hospitals with designated observation units, or where observation status 
care has been receiving attention, may have been more likely to respond to our survey, which may bias our results to reflect the experiences of hospitals experiencing particular successes or challenges with observation status care. A comparison of known hospital characteristics revealed no differences between hospitals that did and did not provide responses to Survey 2 , but other unmeasured differences may exist.

\section{CONCLUSION}

Observation status is assigned using duration of treatment, clinical care guidelines, and level of care criteria, and is defined differently by individual hospitals and payers. Currently, the most widely available setting for pediatric observation status is within a virtual inpatient unit. Our results suggest that the care delivered to observation patients in virtual inpatient units is consistent with care provided to other inpatients. As such, observation status is largely an administrative/billing designation, which does not appear to reflect differences in clinical care. A consistent approach to the assignment of patients to observation status, and treatment of patients under observation among hospitals and payers, may be necessary to compare quality outcomes. Studies of the clinical care delivery and processes of care for short-stay patients are needed to optimize models of pediatric observation care.

Disclosure: Dr Shah received support from the National Institute of Allergy and Infectious Diseases (K01 AI73729), and the Robert Wood Johnson Foundation under its Physician Faculty Scholar Program. The content is solely the responsibility of the authors and does not necessarily represent the official views of the National Institutes of Health. The authors have no other Conflicts of Interest to disclose.

\section{References}

1. Graff LG. Observation medicine: the healthcare system's tincture of time. In: Graff LG, ed. Principles of Observation Medicine. Dallas, TX: American College of Emergency Physicians; 2010. Available at: http://www.acep.org/content.aspx?id=46142. Accessed February 18, 2011.

2. Hoholik S. Hospital 'observation' status a matter of billing. The Columbus Dispatch. February 14, 2011.

3. George J. Hospital payments downgraded. Philadelphia Business Journal. February 18, 2011.

4. Jaffe S. Medicare rules give full hospital benefits only to those with 'inpatient' status. The Washington Post. September 7, 2010.

5. Clark C. Hospitals caught between a rock and a hard place over observation. Health Leaders Media. September 15, 2010.

6. Clark C. AHA: observation status fears on the rise. Health Leaders Media. October 29, 2010.
7. Brody JE. Put your hospital bill under a microscope. The New York Times. September 13, 2010.

8. Medicare Hospital Manual Section 455. Washington, DC: Department of Health and Human Services, Centers for Medicare and Medicaid Services; 2001.

9. Barsuk J, Casey D, Graff L, Green A, Mace S. The Observation Unit: An Operational Overview for the Hospitalist. Society of Hospital Medicine White Paper. May 21, 2009. Available at: http://www. hospitalmedicine.org/Content/NavigationMenu/Publications/White Papers/White Papers.htm. Accessed May 21, 2009.

10. Alpern ER, Calello DP, Windreich R, Osterhoudt K, Shaw KN. Utilization and unexpected hospitalization rates of a pediatric emergency department 23-hour observation unit. Pediatr Emerg Care. 2008; 24(9):589-594.

11. Zebrack M, Kadish H, Nelson D. The pediatric hybrid observation unit: an analysis of 6477 consecutive patient encounters. Pediatrics. 2005;115(5):e535-e542.

12. Macy ML, Kim CS, Sasson C, Lozon MM, Davis MM. Pediatric observation units in the United States: a systematic review. J Hosp Med. 2010;5(3):172-182.

13. Shaw KN, Ruddy RM, Gorelick MH. Pediatric emergency department directors' benchmarking survey: fiscal year 2001. Pediatr Emerg Care. 2003;19(3):143-147.

14. Crocetti MT, Barone MA, Amin DD, Walker AR. Pediatric observation status beds on an inpatient unit: an integrated care model. Pediatr Emerg Care. 2004;20(1):17-21.

15. Marks MK, Lovejoy FH Jr, Rutherford PA, Baskin MN. Impact of a short stay unit on asthma patients admitted to a tertiary pediatric hospital. Qual Manag Health Care. 1997;6(1):14-22.

16. Mace SE, Graff L, Mikhail M, Ross M. A national survey of observation units in the United States. Am J Emerg Med. 2003;21(7): 529-533.

17. Yealy DM, De Hart DA, Ellis G, Wolfson AB. A survey of observation units in the United States. Am J Emerg Med. 1989;7(6):576-580.

18. Balik B, Seitz CH, Gilliam T. When the patient requires observation not hospitalization. J Nurs Admin. 1988;18(10):20-23.

19. Greenberg RA, Dudley NC, Rittichier KK. A reduction in hospitalization, length of stay, and hospital charges for croup with the institution of a pediatric observation unit. Am J Emerg Med. 2006;24(7): 818-821.

20. Listernick R, Zieserl E, Davis AT. Outpatient oral rehydration in the United States. Am J Dis Child. 1986;140(3):211-215.

21. Holsti M, Kadish HA, Sill BL, Firth SD, Nelson DS. Pediatric closed head injuries treated in an observation unit. Pediatr Emerg Care. 2005;21(10):639-644.

22. Mallory MD, Kadish H, Zebrack M, Nelson D. Use of pediatric observation unit for treatment of children with dehydration caused by gastroenteritis. Pediatr Emerg Care. 2006;22(1):1-6.

23. Miescier MJ, Nelson DS, Firth SD, Kadish HA. Children with asthma admitted to a pediatric observation unit. Pediatr Emerg Care. 2005; 21(10):645-649.

24. Krugman SD, Suggs A, Photowala HY, Beck A. Redefining the community pediatric hospitalist: the combined pediatric ED/inpatient unit. Pediatr Emerg Care. 2007;23(1):33-37.

25. Abenhaim HA, Kahn SR, Raffoul J, Becker MR. Program description: a hospitalist-run, medical short-stay unit in a teaching hospital. Can Med Assoc J. 2000;163(11):1477-1480.

26. Hung GR, Kissoon N. Impact of an observation unit and an emergency department-admitted patient transfer mandate in decreasing overcrowding in a pediatric emergency department: a discrete event simulation exercise. Pediatr Emerg Care. 2009;25(3):160-163.

27. Fieldston ES, Hall M, Sills MR, et al. Children's hospitals do not acutely respond to high occupancy. Pediatrics. 125(5):974-981.

28. Macy ML, Stanley RM, Lozon MM, Sasson C, Gebremariam A, Davis MM. Trends in high-turnover stays among children hospitalized in the United States, 1993-2003. Pediatrics. 2009;123(3):996-1002. 